\title{
Study on Surface Asperities in Bakelite-RPC
}

\section{N. Majumdar ${ }^{a *}$, S. Mukhopadhyay ${ }^{a}$, P. Bhattacharya $^{a}$, S. Biswas ${ }^{b}$, S. Bhattacharya ${ }^{a}$, S. Saha ${ }^{a}$, S. Chattopadhyay ${ }^{c}$}

${ }^{a}$ Applied Nuclear Physics Division, Saha Institute of Nuclear Physics, Kolkata-700064, India

${ }^{b}$ Detector laboratory, GSI Helmholtzzentrum fÃijr Schwerionenforschung GmbH, D-64291

Darmstadt, Germany

${ }^{c}$ Experimental High Energy Physics \& Applications Group, Variable Energy Cyclotron Centre, Kolkata, India

E-mail: nayana.majumdar@saha.ac.in,

supratik.mukhopadhyayesaha.ac.in, purba.bhattacharya@saha.ac.in,

S.Biswas@gsi.de, sudeb.bhattacharya@saha.ac.in,

satyajit.saha@saha.ac.in, sub@veccal.ernet.in

The effects of surface roughness and subsequently the surface treatment have been studied numerically for Bakelite-RPC. The models used in the computation are based on the geometrical parameters of Bakelite-RPC prototypes built for INO experiment. The asperities have been modeled following the parameters extracted from the analysis of the measurements of surface roughness of Bakelite plates used in the prototypes. The induced charge on a readout strip has been calculated for the models with asperity on bare Bakelite and surface treated Bakelite. The results have indicated that the treatment of the Bakelite surface with silicone fluid has suppressed the generation of high charges.

PACS: $29.40 . \mathrm{Cs}, 29.40 . \mathrm{Gx}$

XI Workshop on Resistive Plate Chambers and Related Detectors

5-10 February, 2012

Laboratori Nazionali di Frascati dell'INFN - Frascati (Rome) - Italy

* Speaker. 


\section{Introduction}

The choice of Resistive Plate Chamber (RPC) for tracking as well as triggering purposes in many of the modern detection systems is driven by the facts that it can deliver excellent spatial and timing information and it is one of the simplest gaseous detector to construct with comparatively lower material cost. As a result, the chamber has found extensive applications over a wide range of fields, namely high energy physics, astroparticle physics, medical imaging.

Iron CALorimeter (ICAL) is a dedicated setup for studying the atmospheric neutrino in Indiabased Neutrino Observatory (INO) [1] where RPC has been chosen as a prime detection element to be made out of float glass. More than twenty five thousand chambers with dimension of $2 \mathrm{~m} \times 2 \mathrm{~m}$ will be commissioned in ICAL interleaved with iron plates. In parallel to extensive R\&D activities in connection to INO experiment, RPCs made of Bakelite have also undergone detailed investigation. The prime reason for this activity is obviously that the Bakelite as a material is a better choice for building large scale chambers with affordable cost. Furthermore, other requirements of the experiment like consistently high efficiency, long-term stability can also be achieved with the same material. However for some of the grades of Bakelite, a corrective measure like surface treatment may be necessary before achieving the satisfactory performance of the chamber. Coating the surface with an additional material can provide a better surface quality which are found to be beneficial in some cases. The reason is that the irregularities on the material surface arising out of machining and handling are likely to cause distortion in the local field and gain. Depending on their shape and amplitude, the asperities can even be responsible for generating local discharges and related fluctuations which can ultimately degrade the overall performance of the chamber. In case of Bakelite-RPC prototypes built with Bakelites available in India, substantial improvement in efficiency and reduction in counting rate and current have been observed following the application of a thin layer of silicone fluid on Bakelite surface [2].

A simulation work has been pursued to elucidate the role of surface quality in the performance of RPC. The models considered in the calculation have been framed using the geometry of a Bakelite-RPC prototype while the asperities have been modeled based on the data available from the surface profile measurements. Some of the preliminary calculations have already demonstrated that a change of about 5-10\% in the field values at close vicinity of the asperities are possible [3]. In this work, the charge induced on the readout electrode of Bakelite-RPC has been used as a key to explore the effect of applying a coating on the surface of the Bakelite. However, a scaling in the electric field has been required in order to produce a signal of the order of what has been measured actually. The results have justifiably indicated a suppression of higher charges generated due to the asperity on the material surface following the treatment. The detailed calculation and analysis are in progress especially to understand the disagreement in the observation and the calculation and expected to reveal more information in this regard. 


\section{Simulation}

The details of the Bakelite-RPC model used in the simulation along with its geometrical parameters can be found in Ref.[3]. The measurements on the material surface roughness done with a profilometer have been analysed to get representative shape and amplitude of the asperities following a method described in Ref.[3]. In this work, a pyramid-like shape with height $4 \mu \mathrm{m}$ and a square base of $100 \times 100 \mu \mathrm{m}^{2}$ has been considered as a prototype asperity in the model. Calculation of the induced charge has been performed for two cases of Bakelite-RPC. One model contains a surface asperity located centrally on a bare Bakelite plate while the other one consists of a thin layer of silicone fluid of $10 \mu \mathrm{m}$ thickness applied on the Bakelites. The computation has been carried out with Garfield 7.43 [4] with an interface to neBEM 1.8.10 [5] to calculate the induced charge. The transport properties for the gas combination of 95\% TFE with 5\% Isobutane have been obtained from Magboltz 8.9.3 [6]. The signal has been calculated using a model where a fixed number of electrons has been released from a specific location using Runge-Kutta-Fehlberg (RKF) integration technique for simulating drift path.

\section{Results \& Discussion}

The field configuration inside the gas volume of the Bakelite-RPC has been computed at a typical operating voltage $9 \mathrm{kV}$. The field value along the centre of the RPC has been shown in Fig.1. It can be noticed that the field value shoots by 7-8\% near the Bakelite surface where the asperity is located. A close look at the potential contours can indicate the effect of an asperity on

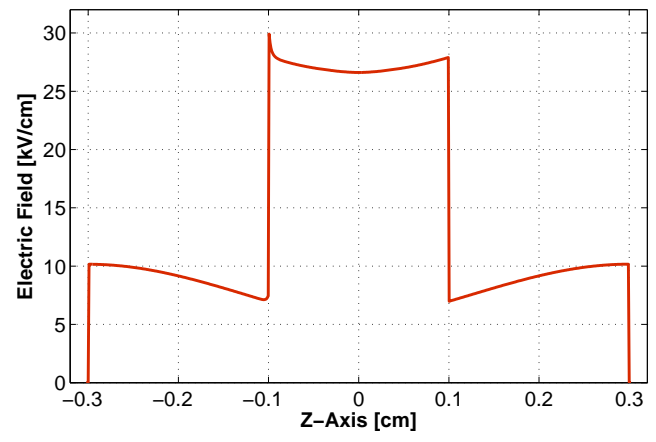

Figure 1: Axial field distribution at $9 \mathrm{kV}$

the field configuration of the chamber at its vicinity. As can be noticed from Fig.2, the asperity can cause a local distortion in the field distribution which may be substantial depending on its shape and amplitude.

While pursuing the calculation of signal induced on the readout strip, it has been noticed that the calculated transport coefficients of TFE+Isobutane(95:5) mixture are insufficient to yield a considerable amplification of the electrons over the operating range of the Bakelite-RPC. The 


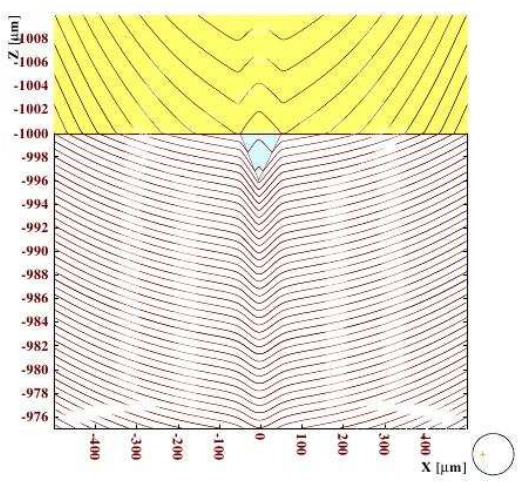

Figure 2: Potential contours near an asperity of amplitude $4 \mu \mathrm{m}$ at $9 \mathrm{kV}$

townsend and attachment coefficients for the said mixture are plotted in Fig. 3 which indicate yield of a few tens of electrons per unit length around $30-35 \mathrm{kV} / \mathrm{cm}$ which is the typical operating regime. It is even lower in case of TFE+Isobutane+SF 6 (95:4.5:0.5) where the last component is added for

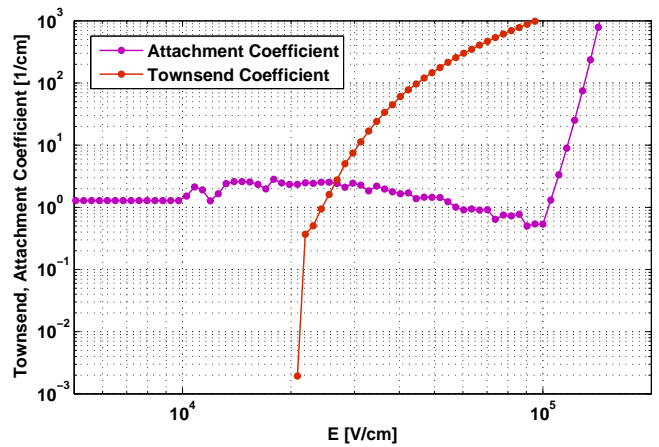

Figure 3: Transport properties of TFE+Isobutane (95:5) mixture

suppressing streamer generated in RPC by escalating the electron attachment capability of the mixture. A comparison of Fig.4 and Fig.5 shows qualitatively the change in the amplification of the electrons when the field rises from $30 \mathrm{kV} / \mathrm{cm}$ to $40 \mathrm{kV} / \mathrm{cm}$ which is also corroborated by the calculation shown in Fig.3. So as to generate a significant signal the electric field has been scaled up from $30 \mathrm{kV} / \mathrm{cm}$ to about $50 \mathrm{kV} / \mathrm{cm}$ by raising the applied voltage to $16 \mathrm{kV}$. This in turn is expected to project the minute signature of the surface treatment, if there is any.

Fig.6 shows a typical plot of induced current on the readout strip due to the movement of electrons inside the gas volume for a Bakelite-RPC with asperity. The induced charge has been calculated by integrating the induced current over a time window and normalized with respect to electronic charge. The charge distributions as generated for two cases of bare and treated Bakelite have been depicted in Fig.7. The upper part representing the bare Bakelite shows two peaks in the distribution. The structure at higher values of charge are likely to arise out of the local field 


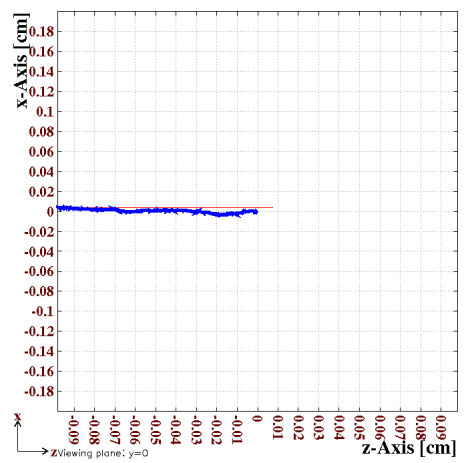

Figure 4: Amplification of an electron released at the centre at $9.86 \mathrm{kV}$

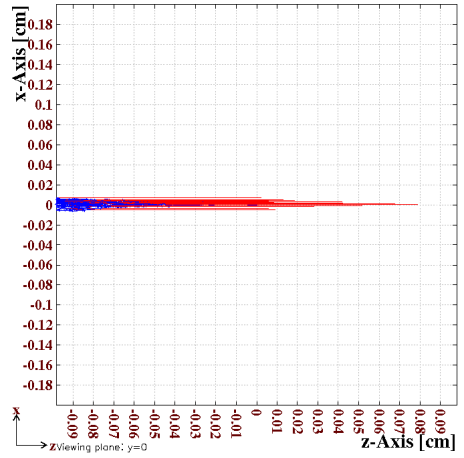

Figure 5: Amplification of an electron released at the centre at $12 \mathrm{kV}$

enhancement caused by the asperity. This observation can be justified with the lower part of the figure which shows the charge distribution when the Bakelite is coated with a layer. This is expected to provide a smooth surface over the Bakelite and thus generate a uniform field configuration where the distribution should be singly peaked.

\section{Conclusion}

The present simulation has indicated that a local fluctuation in the field and gain can be caused by the surface asperities. The structures can induce generation of high charges which may be substantial depending on their shape and amplitude. However, the surface treatment of Bakelite with silicone can improve the performance of the chamber by covering the surface irregularities and thus preventing the localized development of high charges.

The calculation has required a scaled up electric field value inside the RPC volume in order to yield electron amplification substantial enough to project any information. This requires a detailed investigation on the following aspects. Despite the fact the field simulation agrees well with existing analytic and numerical values, it should be checked for errors and compared with measurements. More detailed investigation is required to elucidate the actual field configuration in an 


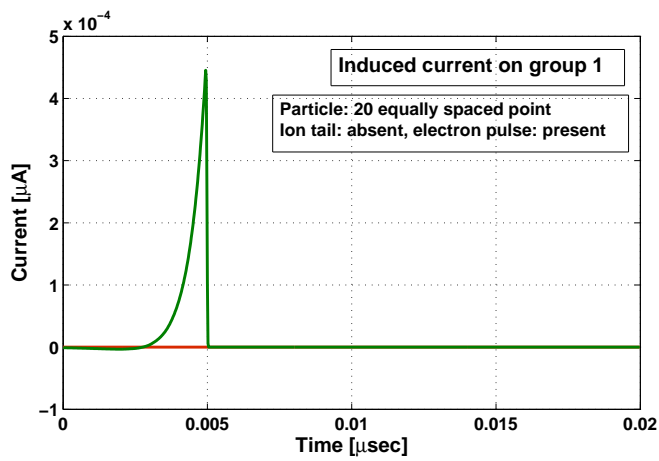

Figure 6: Induced current on a readout strip for bare Bakelite at $16 \mathrm{kV}$
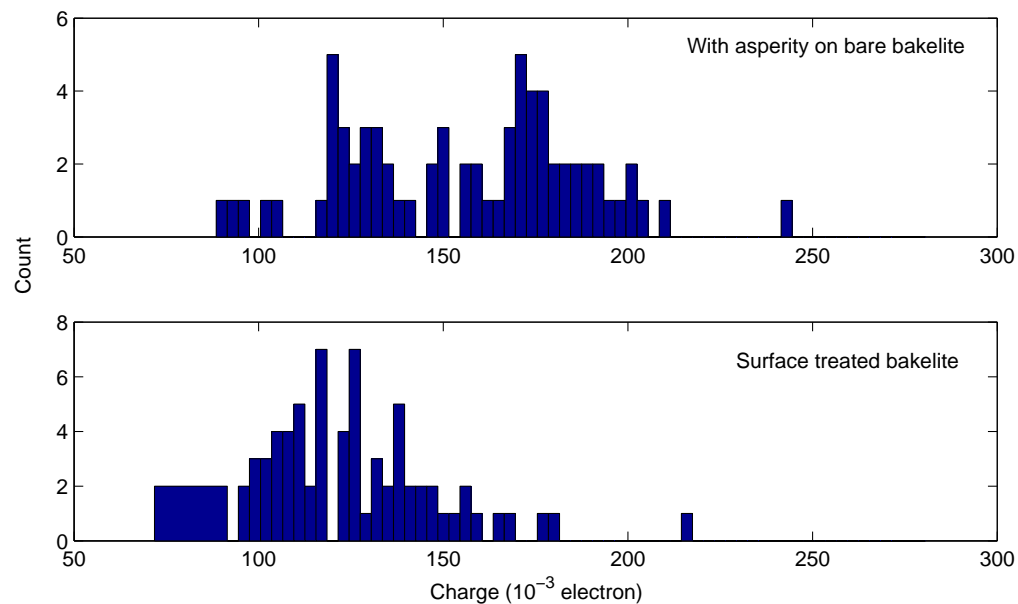

Figure 7: Charge distribution at $16 \mathrm{kV}$ for two Bakelite-RPC models

RPC which seems to be underpredicted by the electrostatic calculation. There can be uncertainties in geometrical parameters like electrical permittivity of various material used in the construction, their actual thickness which are crucial in calculating the field configuration. The transport properties of the gas mixture should also be reviewed with caution since their values indicate very low amplification in the given parameter space.

\section{References}

[1] http://www.imsc.res.in/ ino/

[2] S. Biswas et al., Performances of linseed oil-free bakelite RPC prototypes with cosmic ray muons, NIM A 602 (2009) 749.

[3] S. Biswas et al., Performances of silicone coated high resistive bakelite RPC, NIM A 661 (2012) S94.

[4] http://garfield.web.cern.ch 
[5] http://nebem.web.cern.ch

[6] http://magboltz.web.cern.ch 